\title{
Recursive Model Identification for the Analysis of Cardiovascular Autonomic Modulation During Epileptic Seizure
}

\author{
Quentin Gillardin, Virginie Le Rolle, Anca Nica, Arnaud Biraben, Benoît Martin, Alfredo Hernández \\ Univ Rennes, CHU Rennes, Inserm, LTSI - UMR 1099, F-35000 Rennes, France
}

\begin{abstract}
Significant cardio-respiratory fluctuations are often observed during and after an epileptic seizure event. The mechanisms underlying these acute modifications are considered to be involved in sudden and unexpected death in epilepsy (SUDEP). We hypothesize that these acute events are mediated by specific dynamics of the autonomic nervous system (ANS). However, the evaluation of the ANS during seizures remains particularly challenging, mainly due to the lack of observability. Computational modelling could help to override these limitations, to assess ANS modulation and to evaluate this hypothesis. In this study, we propose and apply a recursive identification algorithm of a system-level model of the autonomic modulation of the sino-atrial node, integrating a Tikhonov regularization, in order to assess sympathetic and parasympathetic activities during ictal tachy-bradycardia events. We evaluate the feasibility of the method on heart rate (HR) data from 4 seizures observed in the same patient. After parameter optimization and identification we were able to reproduce observed HR data with a maximum root mean squared error equals to $1.7 \mathrm{bpm}$. The estimated autonomic series show sympathetic activation and parasympathetic inhibition at the seizure onset, and a massive vagal discharge as the leading factor to ictal bradycardia.
\end{abstract}

\section{Introduction}

The autonomic function is usually altered during and after an epileptic seizure event. These alterations lead, between other effects, to a significant modification of heart rate [1], as well as other cardiac and respiratory functions [2]. Although the hypothesis of an autonomic dysfunction has been already proposed in the context of sudden and unexpected death in epilepsy (SUDEP) [3], the precise underlying mechanisms remain unknown. Autonomic analyses performed on SUDEP patients have shown severe autonomic dysfunctions [4][5]. Animal experiments demonstrated that seizures spread to the brainstem can lead to cardio-respiratory depression and death [6]. It has been also suggested that lethal events are mediated by seizureinduced brainstem depolarization which inactivates auto- nomic control centers [7]. In these cases, the assessment of autonomic nervous system (ANS) activity may be useful to better understand the underlying mechanisms of SUDEP. However, ANS activity is difficult to estimate during seizure. Classical heart rate variability (HRV) analysis tools are not adapted, due to the strong non-stationary character of these series and the suddenness of cardiovascular events. Furthermore, experimental recordings of ANS activity are difficult to acquire.

The objective of this paper is to evaluate the feasibility of a new model-based method to assess sympathetic and parasympathetic dynamics from non-stationary HR series observed during an epileptic seizure event. The method relies on a system-level model of the autonomic modulation of the sinoatrial node, and a recursive identification algorithm. In particular, we propose in this publication a Tikhonov-like regularization approach to enhance the identification process in such an under-determined problem, and provide a method to optimize the regularization parameters. The proposed method was applied to 4 seizures of one patient presenting acute and transient cardiovascular ictal events.

\section{Methods}

\subsection{Clinical Data}

From a clinical research database acquired at $\mathrm{CHU}$ of Rennes with the authorized consent of the patient, we selected a patient with no other pathology but epilepsy, presenting transient seizure-induced acute cardio-respiratory events. Complete polysomnography (PSG) recordings were analyzed to extract 4 seizures with recurrent patterns of ictal tachycardia arising at the onset of the seizure and followed by a transient, significant bradycardia. An example of such events is shown in Fig.1.

\subsection{Data Processing}

RR series were extracted from the available PSG ECGs. Ectopic beats were removed and QRS complex detection were manually corrected to avoid detection errors due to epilepsy-related artefacts. The heart rate series was then 

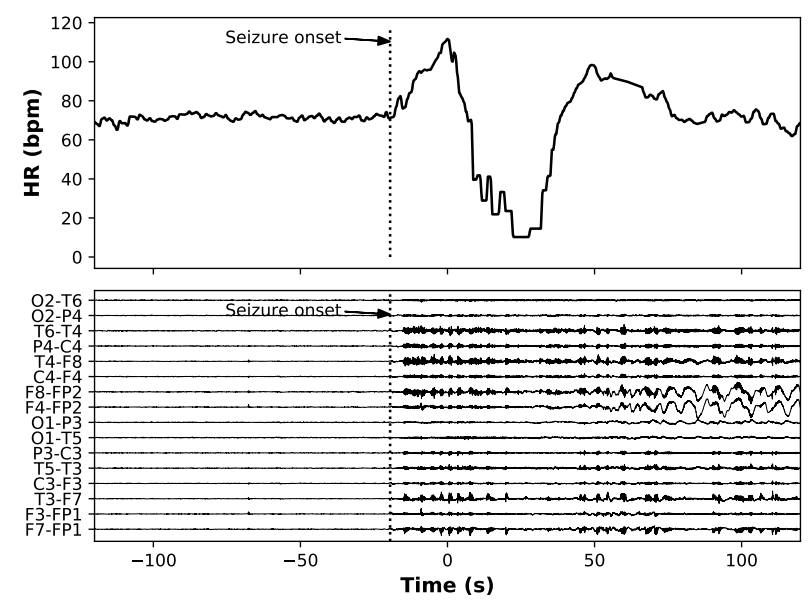

Figure 1. Record showing an acute tachy-bradycardia event occurring during the onset of the seizure (top), and the corresponding cortical activity, presented in longitudinal bipolar setup (bottom).

calculated, interpolated, and resampled at $100 \mathrm{~Hz}$, so as to keep a sufficient temporal resolution for the recursive identification method.

\subsection{Computational Model}

The proposed computational model is focused on efferent nervous activity and is based on a widely used approach [8-10]. Both sympathetic and vagal branches are modeled by first-order filters, characterized by gains $\left(K_{v}\right.$, $\left.K_{s}\right)$ and time constants $\left(T_{v}, T_{s}\right)$ (Fig. 2). The polynomial $P_{H R}$ characterizes the chronotropic response to both sympathetic $\left(F_{s}\right)$ and vagal $\left(F_{v}\right)$ activities [8]. The inputs of the model, $f_{s}$ and $f_{v}$, stand respectively for the sympathetic and parasympathetic efferent activities. These are time-varying variables that aggregate the influence of different sources (blood pressure fluctuations, central modulation, respiration, etc.). We defined the state vector $\vec{S}_{i}=\left(f_{s, i}, f_{v, i}, F_{s, i}, F_{v, i}\right)$ as the representation of the state of the model at each instant $t=i T$.

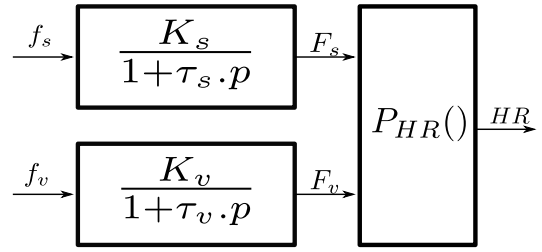

$$
\begin{aligned}
& {\left[K_{s}, K_{v}\right]=[1,0.8]} \\
& {\left[\tau_{s}, \tau_{v}\right]=[6,1.8]} \\
& H R=35+140 . F_{s}-40 . F_{s}^{2}-32 \cdot F_{v}+10 \cdot F_{v}^{2}+20 . F_{s} \cdot F_{v}
\end{aligned}
$$

Figure 2. Block diagram of the system-level model, representing its parameters and main variables.

\subsection{Recursive Identification}

The objective here is to estimate the time series $f_{s}$ and $f_{v}$ that minimize the error between the simulated HR signal $\left(H R_{\text {sim }}\right)$ and the observed HR $\left(H R_{r e f}\right)$. The cost function (1) is calculated on overlapping segments, as:

$$
\begin{gathered}
\epsilon_{i}=\frac{1}{n} \sum_{t_{e}=i T}^{i T+\Omega}\left(H R_{s i m}\left(t_{e}\right)-H R_{r e f}\left(t_{e}\right)\right)^{2}+\Gamma_{s, i}+\Gamma_{v, i} \\
\Gamma_{s, i}=\alpha \sum_{t_{e}=i T-\tau_{s}}^{i T}\left(f_{s}(i T)-f_{s}\left(t_{e}\right)\right)^{2} \\
\Gamma_{v, i}=\beta \sum_{t_{e}=i T-\tau_{v}}^{i T}\left(f_{v}(i T)-f_{v}\left(t_{e}\right)\right)^{2}
\end{gathered}
$$

where $i$ is the step of the identification process, $t_{e}$ is the time elapsed since the onset of the identification period, $\Omega$ is the duration of each interval, $T$ corresponds to the overlap time between each interval, $n$ is the number of samples during $\Omega . \Gamma_{s}$ and $\Gamma_{v}$ are two regularization terms, inspired from the Tikhonov regularization method and introduced to face the under-determination of the problem. They respectively weigh the dynamics of the $i^{t h}$ candidates regarding the past identified values, on a segment of duration equal to their respective time constant $\tau_{s}$ and $\tau_{v}$, so as to privilege smoother solutions. The main procedure of the identification process is described in [9] and depicted in figure 3. At each step $i$, the best set of $\left\{f_{v}, f_{s}\right\}$ was identified within $[0,1]$ by applying a self-adaptive differential evolution algorithm (jDE)[11]. The model was then updated with these inputs to start a new simulation that will generate the new state vector $\vec{S}_{i+1}$ at $t=(i+1) T$. Between the $i^{t h}$ and the $(i+1)^{t h}$ steps of the algorithm, the sliding window was shifted of $T$ seconds and the new step was initialized with $\vec{S}_{i+1}$. This procedure was executed until all the samples of $H R_{r e f}$ had been covered.

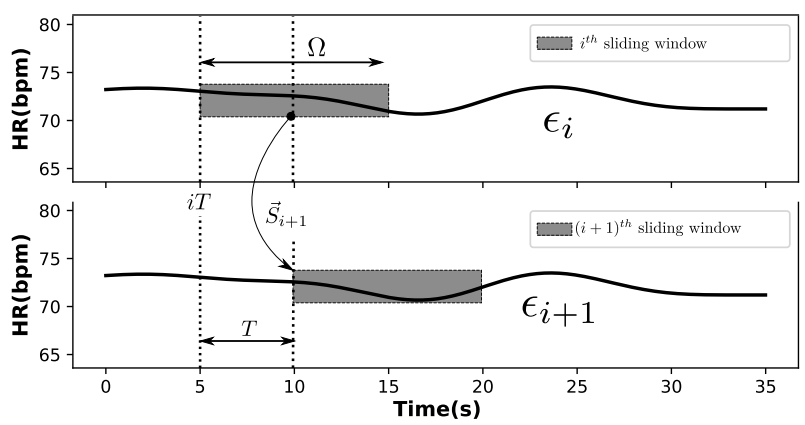

Figure 3. Representation of sliding windows of length $\Omega$ where input values are estimated. For each step, the sliding window is shifted of $T \mathrm{~s}$ and states of the algorithm are set to the latest optimal solution at $t=(i+1) T$. 


\subsubsection{Cost function optimization}

Performance of the identification is highly dependent on parameters $\Omega$ and $T$. In fact, the overlap time $T$ should be able to capture rapid events in $H R_{\text {ref }}$, partly due to the vagal modulation, and acts as the sampling period of the method. The identification interval $\Omega$ should be defined to take into account the slowest dynamics, which could be associated with sympathetic modulation. Constants $\alpha$ and $\beta$, associated with regularization terms, also had an impact on the results. In order to find optimal identification results, an exhaustive exploration of $\Omega, T, \alpha$ and $\beta$ was performed by executing the recursive identification procedure for each combination of these identification parameters within a discrete grid. From each execution, a simulated heart rate $\widehat{H R}_{\text {sim }}$ was constructed from state vectors $\vec{S}$. The optimal values of $\Omega$ and $T$ were chosen in order to minimize the root mean squared error (RMSE) between $\widehat{H R}_{\text {sim }}$ and $H R_{\text {ref }}$ (eq.4) where $t_{s}$ is the sampled time, $N$ is the total number of samples and $t_{t o t}$ corresponds to signal duration.

$$
R M S E=\sqrt{\frac{1}{N} \sum_{t_{s}=0}^{t_{\text {tot }}}\left(\widehat{H R}_{\text {sim }}\left(t_{s}\right)-H R_{r e f}\left(t_{s}\right)\right)^{2}}
$$

Parameters $\alpha$ and $\beta$ were selected in such a way that the identification procedure gives a realistic autonomic activity without degrading the efficiency of the identification process.

\section{Results and Discussion}

The cost function optimization process was applied to the four available HR series, corresponding to four epileptic seizures of a given patient. Figure 4 illustrates an example of RMSE values calculated for different values of $T$ and $\Omega$ for one seizure. Results showed the existence of a global minimum in the range of parameters delimited by time constants of the model. We chose $\alpha=\beta=0.1$. These weights give both the ability to the process to fit the input data, and to give a realistic sympathetic and parasympathetic identified activities [12].

Figure 5 shows the results of the recursive identification procedure on each of the four seizures analyzed. Results show, i) the comparison between simulated and observed HR signals, ii) the dynamics of $f_{s}$ and $f_{v}$ as identified with the proposed approach and iii) the optimal values of $T$ and $\Omega$ for each case. A close match was observed between simulated and observed signals, with an average RMSE of $0.52 b p m$. In the case of seizure 2, the error is three times greater than this mean value. The identification process didn't succeed to fit the bradycardia, and the identified ANS activities lead to saturation of the model.

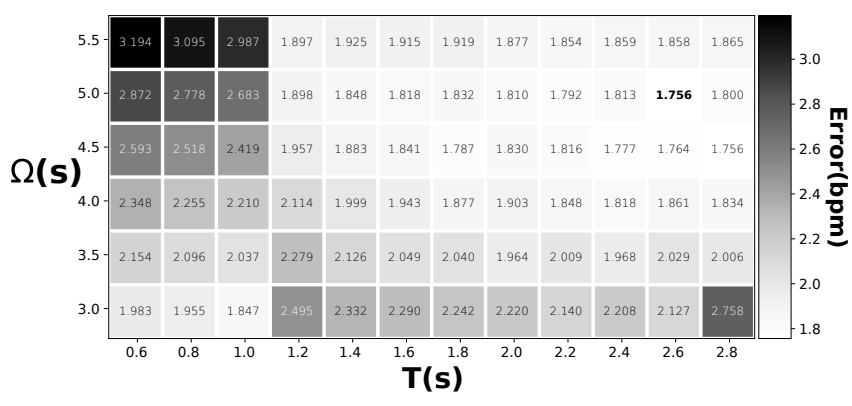

Figure 4. Example heat map showing RMSE calculated for different values of $\mathrm{T}$ and $\Omega$, when fixing $\alpha=\beta=0.1$.

This emphasise that $P_{H R}$ is unsuitable for the analysis of this acute event. A patient-specific description of the chronotropic response to ANS stimulation might be used in this case. Concerning the identified sympathetic and parasympathetic activities (Fig.5), for each seizure, our method is able to reproduce the sympathetic activation and parasympathetic inhibition induced by seizure [12]. Also, our model suggests that transient bradycardia following the seizure-induced tachycardia may be due to a massive vagal activation and a sympathetic withdrawal. This results are in accordance with recent animal experiments in the field suggesting that bradycardia preceding SUDEP is due to a significant vagal discharge [13].

\section{Conclusions}

In this paper we proposed a model-based method relying on a recursive stochastic algorithm to estimate ANS dynamics from non-stationary, observed HR series. We proposed a method to better condition this process, to reproduce $\mathrm{HR}$, and to estimate realistic ANS activity. Results warrant further investigation to enhance identification of ANS activity, especially to study the underlying mechanisms of SUDEP.

\section{Acknowledgements}

This work was supported by the French National Research Agency (ANR) (project AdaptVNS) and the French Regional Council of Brittany (Project CRAMSIE).

\section{References}

[1] Russell AE. Cessation of the pulse during the onset of epileptic fits,. with remarks on the mechanism of fits. The Lancet 1906;168(4325):152-154.

[2] Baumgartner C, Koren J, Britto-Arias M, Schmidt S, Pirker S. Epidemiology and pathophysiology of autonomic seizures: a systematic review. Clinical Autonomic Research apr 2019;29(2):137-150.

[3] Nashef L, So EL, Ryvlin P, Tomson T. Unifying the defi- 

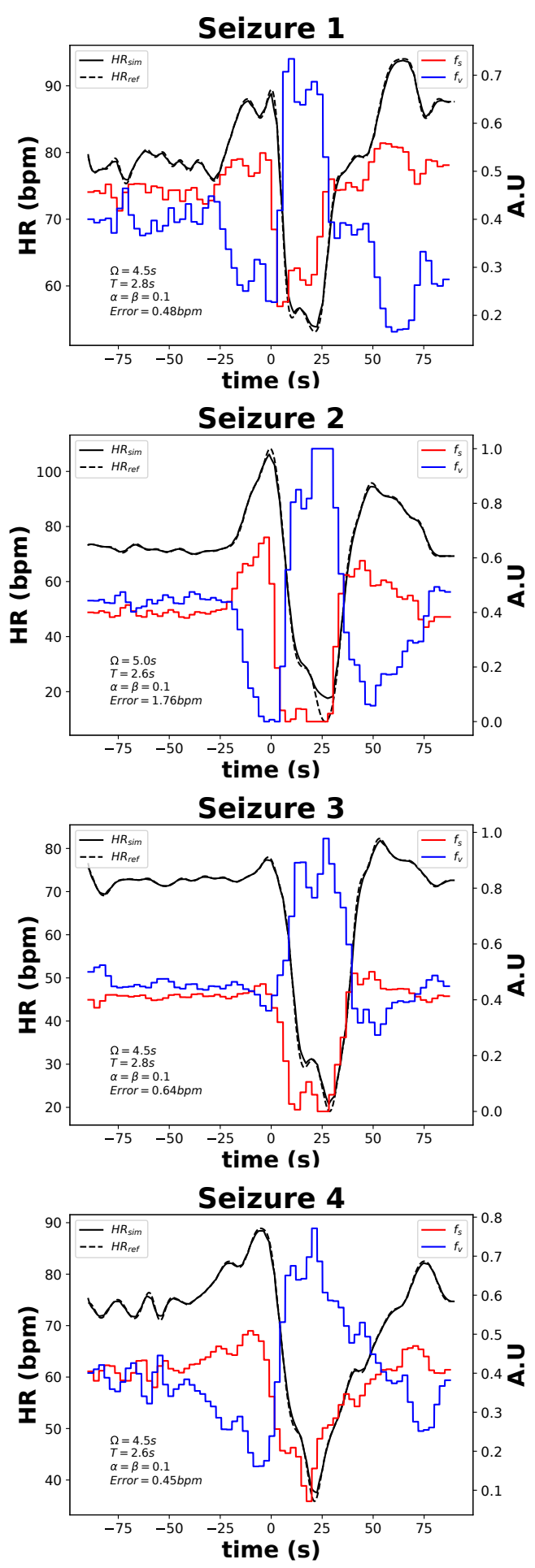

Figure 5. Identification results over four seizures showing the reference HR signal (black dashed), the simulated HR signal (solid black) and the identified sympathetic (red) and vagal (blue) activities. nitions of sudden unexpected death in epilepsy. Epilepsia 2012;53(2):227-233.

[4] Jeppesen J, Fuglsang-Frederiksen A, Brugada R, Pedersen B, Rubboli G, Johansen P, Beniczky S. Heart rate variability analysis indicates preictal parasympathetic overdrive preceding seizure-induced cardiac dysrhythmias leading to sudden unexpected death in a patient with epilepsy. Epilepsia jul 2014;55(7):e67-e71.

[5] Myers KA, Bello-Espinosa LE, Symonds JD, Zuberi SM, Clegg R, Sadleir LG, Buchhalter J, Scheffer IE. Heart rate variability in epilepsy: A potential biomarker of sudden unexpected death in epilepsy risk. Epilepsia jul 2018; 59(7):1372-1380.

[6] Lertwittayanon W, Devinsky O, Carlen PL. Cardiorespiratory depression from brainstem seizure activity in freely moving rats. Neurobiology of Disease feb 2020;134(October 2019): 104628.

[7] Aiba I, L.Noebels J. Spreading depolarization in the brainstem mediates sudden cardiorespiratory arrest in mouse sudep models. Science Translational Medicine 2015; 14(11):871-882.

[8] Lu K, Clark JW, Ghorbel FH, Ware DL, Bidani A. A human cardiopulmonary system model applied to the analysis of the valsalva maneuver. Am J Physiol Hear Circ Physiol 2001;281(6 50-6):2661-2679.

[9] Le Rolle V, Beuchee A, Praud JP, Samson N, Pladys P, Hernández AI. Recursive identification of an arterial baroreflex model for the evaluation of cardiovascular autonomic modulation. Comput Biol Med nov 2015;66:287294.

[10] Calvo M, Le Rolle V, Romero D, Béhar N, Gomis P, Mabo P, Hernández AI. Recursive model identification for the analysis of the autonomic response to exercise testing in brugada syndrome. Artificial Intelligence in Medicine 2019;97(October):98-104.

[11] Biscani F, Izzo D, Jakob W, GiacomoAcciarini, Mrtens M, C M, Mereta A, Kaldemeyer C, Lyskov S, Corlay S, acxz, Pritchard B, Manani K, Mabille J, Acciarini $\mathrm{G}$, Webb O, Huebl A, v. Looz M, Lpez-Ibez M, jakirkham, Lee J, hulucc, polygon, Travers J, Jordan J, Smirnov I, Nguyen H, Lema F, O'Leary E, Mambrini A. esa/pagmo2: pagmo 2.15.0, April 2020. URL https://doi.org/10.5281/zenodo.3738182.

[12] Novak V, Reeves AL, Novak P, Low PA, Sharbrough FW. Time-frequency mapping of rr interval during complex partial seizures of temporal lobe origin. J Auton Nerv Syst sep 1999;77(2-3):195-202.

[13] Schilling WP, McGrath MK, Yang T, Glazebrook PA, Faingold CL, Kunze DL. Simultaneous cardiac and respiratory inhibition during seizure precedes death in the $\mathrm{dba} / 1$ audiogenic mouse model of sudep. PLoS One 2019; 14(10):1-13.

Address for correspondence:

Alfredo Hernández : alfredo.hernandez@inserm.fr, LTSI - INSERM U1099. Universit de Rennes 1. Campus de Beaulieu. 35042. Rennes France 